\title{
The Protective Effects of Galangal (Alpinia galanga) Against Cyclophosphamide-Induced Cardiotoxicity in Rats
}

\author{
Amjad A. Kokandy and Hanan S. Althagafy \\ Biochemistry Department, Faculty of Science, University of Jeddah, Jeddah, Saudi Arabia.
}

\begin{abstract}
Cyclophosphamide (CP) is a nitrogen mustard alkylating agent used in cancer treatment and immunosuppressant therapy. The clinical use of CP is limited by its dose-dependent cardiotoxicity. The aim of this study was to investigate the cardioprotective effect of galangal (Alpinia galanga) rhizome extract against CP-induced cardiotoxicity in rats. Forty Wistar rats were used, and they were divided into four groups of 10 rats each. Group 1: the control group was administered with saline; group II received CP $(50 \mathrm{mg} / \mathrm{kg} / \mathrm{d}$, intraperitoneally) for 3 days; groups III received $200 \mathrm{mg} / \mathrm{kg} /$ day galangal, orally for 14 days and groups IV was pretreated with $200 \mathrm{mg} / \mathrm{kg} /$ day galangal, orally for 14 days followed by CP $(50 \mathrm{mg} / \mathrm{kg} /$ day, i.p. $)$ for 3 days. Results showed that animals exposed to CP shown a significant decrease in an activities of mitochondrial respiratory chain enzymes complexes, reduced antioxidant enzymes activities, and reduced glutathione levels. Furthermore, CP treatment resulted in an increase in the levels of cardiac serum markers, and lipids. However, the administration of galangal attenuated the biochemical changes induced by CP. This study showed a beneficial effect of galangal in the prevention of CP-induced cardiotoxicity in experimental animals.
\end{abstract}

Keywords: cardioprotective; methanolic extract; mitochondrial complexes; cardiac biomarkers; antioxidant activity 